\title{
Torque-sensorless Control for a Powered Exoskeleton Using Highly Back-drivable Actuators
}

\author{
Yoshiki Kanai \\ Department of Electrical and Computer Engineering \\ Yokohama National University \\ Tel: +81-45-339-4174 \\ Email: kanai-yoshiki-wr@ynu.jp
}

\author{
Yasutaka Fujimoto \\ Department of Electrical and Computer Engineering \\ Yokohama National University \\ Tel: +81-45-339-4174 \\ Email: fujimoto@ynu.ac.jp
}

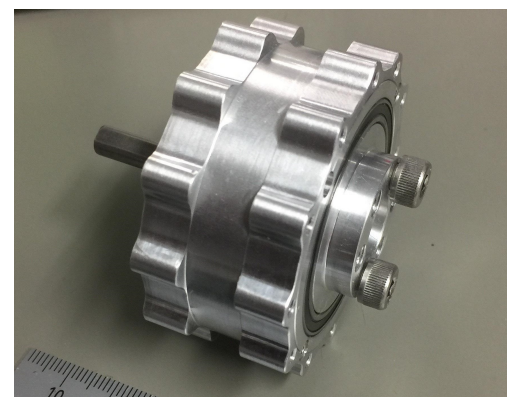

\begin{abstract}
In recent years, most countries have experienced a shift toward aging population, leading to a decline in the working population; thus, an increased physical burden on workers results. The use of powered exoskeletons is one approach to reduce physical stresses on the bodies of workers and elderly people. However, conventional powered exoskeletons have many sensors and actuators mounted on them, resulting in problems associated with their high price, high weight, etc. In this paper, we introduce a new high-efficiency reduction gearbox, which is being researched and developed in our laboratory as a substitute for conventional actuators, and present the results of its attempted assist control without a torque sensor. It was found that the user's torque can be estimated with high accuracy without using a torque sensor by using a highly efficient gearbox with $1 / 102.14$ reduction ratio. In addition, we performed motion identification using a nonlinear support vector machine with the aim of assist control with reduced discomfort on the body by suppressing vibrations.
\end{abstract}

\section{INTRODUCTION}

With a shift toward the aging population in developed countries, there has been a decline in the size of working populations, which is a serious problem. Due to the decline in the number of workers, the workload of the elderly and weak workers will increase; hence, their physical burden as a consequence. Especially in industries such as transportation, agriculture and nursing care, there is also the possibility that such workers will suffer lower back pain, causing a hindrance to their ability to work. Therefore, many assistive robots [1] [6] have been developed. A type of assistive robot known as a "Powered exoskeleton" can be used to alleviate the physical burden on these workers, and research on this subject has advanced greatly [3]-[6]. A powered exoskeleton is a robot that can be directly fitted to the human body, to assist its natural physical functions and can be used for the purpose of reducing the burden of physical labor. However, because many sensors and actuators are mounted on a conventionally powered exoskeleton, various problems related to its associated high price, high weight, difficulty in attaching and detaching it to the body, etc., are emerging. These are some of the reasons why the established powered exoskeleton is not popular; thus, there are demands for a safer, high-performance powered exoskeleton with fewer sensors.

Currently, one of the widely used types of actuators is a motor with reduction gearboxes [3]-[6]. However, the harmonic gearboxes with $1 / 100$ reduction ratio used in many robots have low back-drivability. Robots that make direct physical contact with people, such as powered exoskeletons, need to be safely and flexibly driven against the external forces. Therefore, various methods for improving back-drivability have been developed. One such method involves the detection of external forces on the robot via torque sensors [4]-[6]. This method is capable of accurate force control because of the accurate measurements acquired from the torque sensors. Another method is to insert elastic bodies as springs into the actuator, forming a highly flexible "series elastic actuator" [6]-[8]. However, in both methods, the systems are altogether costly due to the requirement of additional elements, such as the aforementioned torque sensors and elastic elements.

To solve the problem of low back-drivability of the reduction gearbox, we have developed a highly efficient gearbox [9] named Bilateral Drive, as shown in Fig. 1. This reduction gearbox is a specially-configured $3 \mathrm{~K}$ compound planetary gearbox by maximizing the forward driving efficiency. In our developed gearbox the forward driving efficiency is $89.9 \%$ and the backward driving efficiency is $89.2 \%$. Further, the reverse start torque is only $0.016 \mathrm{~N} \cdot \mathrm{m}$; therefore, this gearbox can be back-driven with a very small torque. The goal of this research was to build a high-performance powered exoskeleton that had an improved back-drivability of the gearbox and could estimate and control the motion of the user without the requirement of additional elements. 


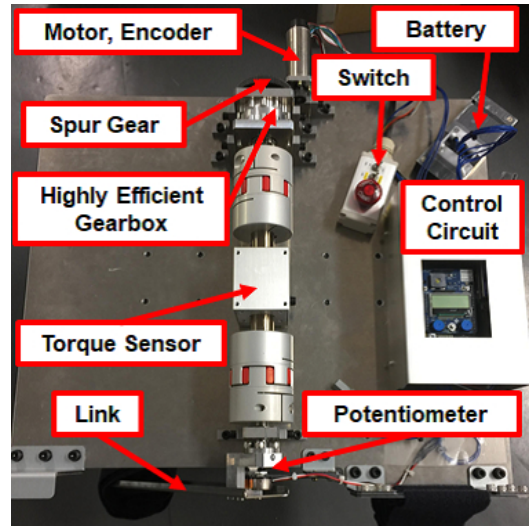

Fig. 2: Structure of experimental machine.

In this paper, we present the control model of the experimental machine, and methods for torque estimation and motion identification using a nonlinear support vector machine (SVM). Finally, we compare estimated values with the sensor values obtained from the conducted experiments.

\section{Control MOdel}

\section{A. Structure of the experimental machine}

In our research, we used a uni-axial articulated link mechanism with one leg as the experimental machine, as shown Fig. 2. During machine operation, the shin was fixed to the link and the potentiometer measured the knee joint angle. The signal output of the potentiometer was $0^{\circ}$ when the link was parallel to the ground and was $-90^{\circ}$ when it was perpendicular to the ground. The torque sensor was used for evaluation and not for control. The control program ran in the microcomputer in the control circuit. The signal of the sensors was input to the control circuit, the motor reference current value was subsequently determined, and the motor was then driven by the motor driver.

\section{B. Moment of inertia}

We expressed double inertial systems as a control model of experimental machine. The equations of motion for the inertia systems are as follows:

$$
\begin{aligned}
J_{M} \ddot{\theta}_{M} & =\tau_{M}-\frac{1}{N} \tau_{s}-\tau_{f}, \\
J_{L} \ddot{\theta}_{L} & =\tau_{s}-\tau_{d},
\end{aligned}
$$

where $J_{M}$ and $J_{L}$ are the moments of inertia of the motor side and load side, respectively; $\ddot{\theta}_{M}$ and $\ddot{\theta}_{L}$ are the angular accelerations of the motor side and load side, respectively; $N(\gg 1)$ is total reduction ratio; $\tau_{M}$ is motor torque; $\tau_{s}$ is shaft torque; $\tau_{f}$ is friction torque of motor side; and $\tau_{d}$ is external torque of the load side. Although the leg link is attached to the experimental machine, it operates simultaneously with the leg of the user, thus, a gravity torque is included in the external torque. The equation for the shaft torque is given as follows:

$$
\tau_{s}=K_{s}\left(\frac{\theta_{M}}{N}-\theta_{L}\right),
$$

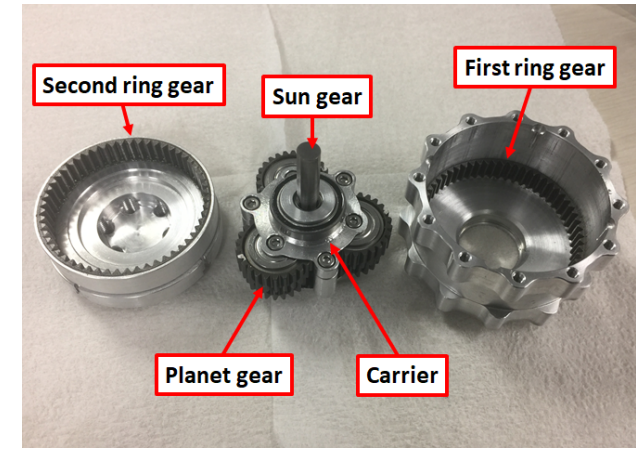

Fig. 3: Disassembled model of bilateral drive gear.

where $K_{s}$ is stiffness. The moments of inertia of motor side and load side, respectively, are given as follows:

$$
\begin{aligned}
J_{M} & =J_{m}+J_{s 1}+J_{s 2}\left(\frac{1}{N_{s}}\right)^{2}, \\
J_{L} & =J_{g}+J_{\text {Torque }}+J_{\text {Couple }}+J_{\text {Link }},
\end{aligned}
$$

where, for the subscript of the moment of inertia $J, m$ is motor, $s 1$ is pinion gear, $s 2$ is spur gear, $g$ is highly efficient gearbox, "Torque" is torque sensor, "Couple" is coupling, and "Link" is leg link. $N_{s}$ is the reduction ratio of spur gear. We derived the total equivalent moment of inertia seen from the load side via kinetic relations. A disassembled model of the highly efficient gearbox is shown in Fig. 3 and its stick diagram is shown in Fig. 4. In Fig. $4, Z_{s}$ is sun gear, $Z_{p 1}$ and $Z_{p 2}$ are first and second planet gear, and $Z_{r 1}$ and $Z_{r 2}$ are first and second ring gear. The equations of speed between the gears are as follows:

$$
\begin{aligned}
r_{s} \omega_{s} & =\left(r_{s}+r_{p 1}\right) \omega_{c}-r_{p 1} \omega_{p 1}, \\
r_{r 1} \omega_{r 1} & =\left(r_{r 1}-r_{p 1}\right) \omega_{c}+r_{p 1} \omega_{p 1}, \\
r_{r 2} \omega_{r 2} & =\left(r_{r 1}-r_{p 2}\right) \omega_{c}+r_{p 2} \omega_{p 2},
\end{aligned}
$$

where $r$ is gear radius and $\omega$ is the gear speed. For the subscripts, $s$ is sun gear, $p 1$ is first planet gear, $p 2$ is second planet gear, $r 1$ is first ring gear, $r 2$ is second ring gear, and $c$ is carrier. Because the first and second planet gears are connected, we can say that $\omega_{p}=\omega_{p 1}=\omega_{p 2}$. Additionally, $\omega_{r 1}=0$ because the first ring gear is fixed. The, speed ratio equations are given as follows.

$$
\begin{aligned}
\frac{\omega_{s}}{\omega_{r 2}} & =\frac{1+I_{1}}{1-I_{2}}, \\
\frac{\omega_{c}}{\omega_{r 2}} & =\frac{1}{1-I_{2}}, \\
\frac{\omega_{p}}{\omega_{r 2}} & =\frac{1-r_{r 1} / r_{p 1}}{1-I_{2}},
\end{aligned}
$$

where

$$
\begin{aligned}
I_{1} & =\frac{r_{r 1}}{r_{s}}, \\
I_{2} & =\frac{r_{r 1} r_{p 2}}{r_{r 2} r_{p 1}} .
\end{aligned}
$$




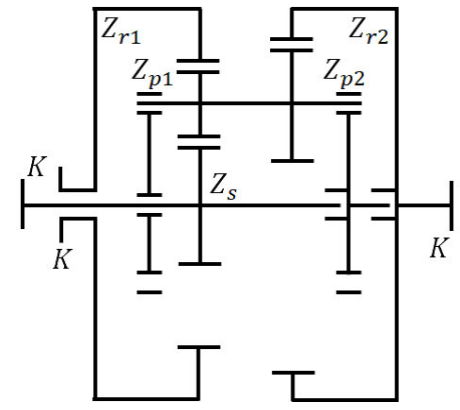

Fig. 4: Stick diagram of $3 \mathrm{~K}$ compound planetary gearbox.

TABLE I: Parameters of the experimental machine

\begin{tabular}{ccc}
\hline \hline & Contents & Value \\
\hline$r_{s}$ & pitch circle radius of sun gear & $2.79[\mathrm{~mm}]$ \\
$r_{p 1}$ & pitch circle radius of first planet gear & $10.4[\mathrm{~mm}]$ \\
$r_{p 2}$ & pitch circle radius of second planet gear & $8.77[\mathrm{~mm}]$ \\
$r_{r 1}$ & pitch circle radius of first ring gear & $23.5[\mathrm{~mm}]$ \\
$r_{r 2}$ & pitch circle radius of second ring gear & $21.9[\mathrm{~mm}]$ \\
$r_{c}$ & radius of carrier & $13.1[\mathrm{~mm}]$ \\
$m_{p}$ & mass of planet gear & $17.9[\mathrm{~g}]$ \\
$k$ & number of planet gears & 3 \\
$J_{m}$ & motor inertia & $3.33 \times 10^{-6}\left[\mathrm{~kg} \cdot \mathrm{m}^{2}\right]$ \\
$J_{s 1}$ & pinion inertia (spur gear) & $5.54 \times 10^{-7}\left[\mathrm{~kg} \cdot \mathrm{m}^{2}\right]$ \\
$J_{s 2}$ & gear inertia (spur gear) & $1.23 \times 10^{-4}\left[\mathrm{~kg} \cdot \mathrm{m}^{2}\right]$ \\
$J_{g}$ & gearbox inertia & $2.95 \times 10^{-3}\left[\mathrm{~kg} \cdot \mathrm{m}^{2}\right]$ \\
$J_{\text {Torque }}$ & torque sensor inertia & $6.60 \times 10^{-5}\left[\mathrm{~kg} \cdot \mathrm{m}^{2}\right]$ \\
$J_{\text {Couple }}$ & coupling inertia & $8.0 \times 10^{-4}\left[\mathrm{~kg} \cdot \mathrm{m}^{2}\right]$ \\
$J_{\text {Link }}$ & link inertia & $1.19 \times 10^{-2}\left[\mathrm{~kg} \cdot \mathrm{m}^{2}\right]$ \\
$N_{s}$ & reduction ratio of spur gear & 4.0 \\
$N_{g}$ & reduction ratio of gearbox & $715 / 7 \approx 102.14$ \\
$J_{M}$ & motor side inertia & $1.16 \times 10^{-5}\left[\mathrm{~kg} \cdot \mathrm{m}^{2}\right]$ \\
$J_{L}$ & load side inertia & $1.61 \times 10^{-2}\left[\mathrm{~kg} \cdot \mathrm{m}^{2}\right]$ \\
\hline \hline
\end{tabular}

The equivalent moment of inertia of the highly efficient gearbox as seen from the output side is expressed by the sum of the moments of inertia about the center axis of each gear multiplied by the square of the speed ratio. Additionally, because of carrier and planet gears are connected, it is necessary to add the moment of inertia of the planet gears about the center axis of carrier to the moment of inertia of the carrier $J_{c}$. This moment of inertia, $J_{c}^{\prime}$, is derived as follows:

$$
J_{c}^{\prime}=J_{c}+k m_{p} r_{c}^{2},
$$

where $J_{p}$ is the moment of inertia of the planet gear and $m_{p}$ is the mass of the planet gear. The total equivalent moment of inertia, $J_{g}$, is given as follows:

$$
J_{g}=J_{r 2}+k J_{p}\left(\frac{\omega_{p}}{\omega_{r 2}}\right)^{2}+J^{\prime}{ }_{c}\left(\frac{\omega_{c}}{\omega_{r 2}}\right)^{2}+J_{s 1}\left(\frac{\omega_{s}}{\omega_{r 2}}\right)^{2}
$$

where, $J_{r 2}$ is the moment of inertia of the second ring gear, $k$ is the number of planet gears, and $J_{s 1}$ is the moment of inertia of the sun gear. The parameters of the experimental machine are shown in Table I.

\section{Friction compensation}

We modeled the friction torque $\tau_{f}$ as follows.

$$
\tau_{f}=D \dot{\theta}_{M}+\tau_{c} \operatorname{sgn}\left(\dot{\theta}_{M}\right),
$$

where $D$ is the viscous friction coefficient and $\tau_{c}$ is the coulomb friction torque. We use feedforward compensation [10] from the friction torque using the friction model, as shown in Fig. 5.

\section{Torque estimation}

We used a multi-encoder-based disturbance observer (MEDOB) [11] as the external torque estimation method in the double inertia system. Using MEDOB, the torque $\hat{\tau}_{d}$ is estimated by the following equation:

$$
\begin{aligned}
\hat{\tau}_{d}= & \frac{g}{s+g}\left(N \tau_{\text {ref }}+N g J_{M} \dot{\theta}_{M}+g J_{L} \dot{\theta}_{L}\right) \\
& -N g J_{M} \dot{\theta}_{M}-g J_{L} \dot{\theta}_{L},
\end{aligned}
$$

where $\tau_{\text {ref }}$ is torque reference value of the motor and $g$ is the cutoff frequency of the MEDOB. In the MEDOB, it is possible to estimate the external torque without using the stiffness $K_{s}$ in the double inertial system. After the experiment, we compared the estimated torque and the output torque value of the torque sensor. In addition, assist control was performed where this estimation was fed back to the input torque and was used to drive the motor in the same direction as the wearer's direction of motion. The torque reference value of the motor $\tau_{\text {ref }}$ is obtained as follows:

$$
\tau_{\text {ref }}=-\frac{K_{\text {Assist }}}{N} \hat{\tau}_{d},
$$

where, $K_{\text {Assist }}$ is the assist gain. By multiplying the assist gain at the time of feedback of the estimated torque value, the ratio of the assist torque to be given to the force applied by the user can be determined. The overall control model is shown in Fig. 5.

\section{Motion IDENTIFICATION METHOD}

To reduce discomfort inside the powered exoskeleton when performing assist control, an appropriate and instantaneous output of the exoskeleton in response to the motion of the user is required. For that purpose, information of the user's intended motion is required. Because the experimental machine is restricted to the bending and stretching of the knee in the state of sitting, the motion state can be divided into two classes: "in operation" and "stopped." The values for the estimated torque and the knee joint angular velocity are used for discriminating between the classes, and when both values are near 0 , the motion is classified "stopped," otherwise it is classified as "in operation." In this paper, a nonlinear SVM [12][11] is used as a method of identification of motion intention. An SVM uses machine learning to generate items that fall into one of two classes with linear input elements. 


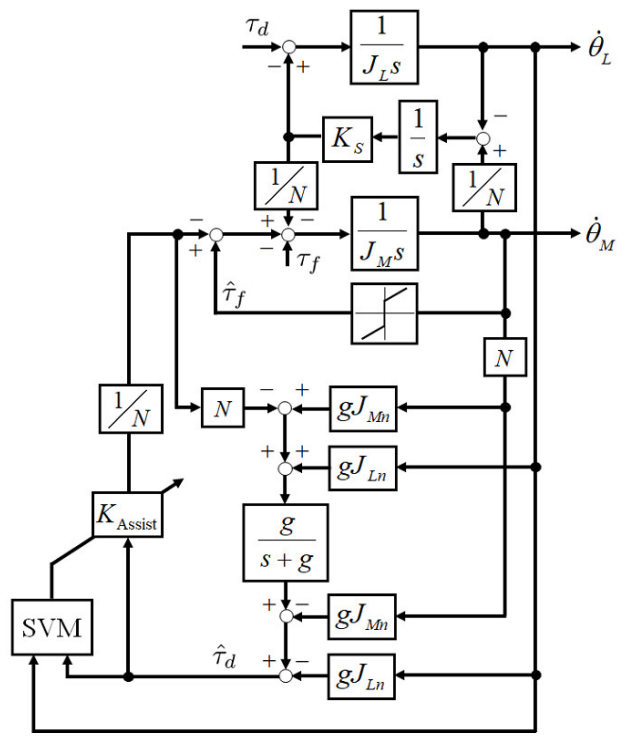

Fig. 5: Control model.

TABLE II: Parameters of the friction model measurement

\begin{tabular}{ccc}
\hline \hline & Contents & Value \\
\hline$\Delta t$ & sampling time & $500[\mu \mathrm{s}]$ \\
$K_{T}$ & torque constant & $13.6 \times 10^{-3}[\mathrm{~N} \cdot \mathrm{m} / \mathrm{A}]$ \\
$J_{M}^{\prime}$ & motor side equivalent inertia & $1.16 \times 10^{-5}\left[\mathrm{~kg} \cdot \mathrm{m}^{2}\right]$ \\
$g$ & cutoff frequency of DOB & $500[\mathrm{rad} / \mathrm{s}]$ \\
$g_{d}$ & cutoff frequency of pseudo derivation & $100[\mathrm{rad} / \mathrm{s}]$ \\
$g_{\text {encoder }}$ & cutoff frequency of LPF for encoder & $62.8[\mathrm{rad} / \mathrm{s}]$ \\
$N$ & total reduction gear ratio & 408.6 \\
\hline \hline
\end{tabular}

\section{EXPERIMENTS}

\section{A. Friction model measurement}

First, we created a friction model of the experimental machine for use in friction compensation. We then measured the motor-side load torque when the motor was driven at a constant angular speed. The control system model was a singular inertial system model as seen from the motor side. For the constant angular velocity rotation, a proportionalintegral derivative (PID) controller was used to control the velocity and the motor-side load torque was estimated by the disturbance observer (DOB) [14]. Additionally, to ignore the influence of the gravity torque, the link was removed. We plotted the average of the load torques when the motor reached a constant angular speed rotation and found line of best fit using the least squares fitting method for each the forward and reverse rotation. The measurement parameters and results are shown in Table II and Fig. 6, respectively. Because the characteristics were different depending on the direction of rotation, in the complete model, different friction models were also made depending on the rotation direction. Furthermore, we applied a "dead zone" to stop the operation near $0 \mathrm{rad} / \mathrm{s}$. The parameters of the friction model obtained by the least squares fitting method are shown in Table III.

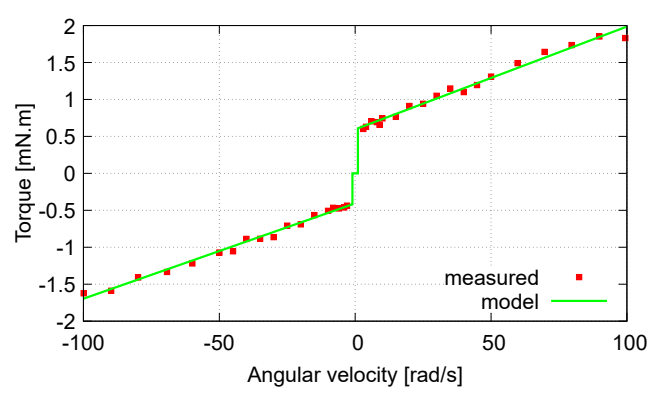

Fig. 6: The friction model.

TABLE III: Parameters of the friction model.

\begin{tabular}{c|cc}
\hline \hline conditions & $D \times 10^{-5}[\mathrm{~N} \cdot \mathrm{m} \cdot \mathrm{s} / \mathrm{rad}]$ & $\tau_{c} \times 10^{-4}[\mathrm{~N} \cdot \mathrm{m}]$ \\
\hline$\dot{\theta}_{M}>0$ & 1.39 & 5.96 \\
$\dot{\theta}_{M}<0$ & 1.29 & 4.06 \\
\hline \hline
\end{tabular}

TABLE IV: Parameters of the experiment

\begin{tabular}{ccc}
\hline \hline & Contents & Value \\
\hline$\Delta t$ & sampling time & $1.0[\mathrm{~ms}]$ \\
$J_{M}$ & motor side inertia & $1.16 \times 10^{-5}\left[\mathrm{~kg} \cdot \mathrm{m}^{2}\right]$ \\
$J_{L}$ & load side inertia & $1.61 \times 10^{-2}\left[\mathrm{~kg} \cdot \mathrm{m}^{2}\right]$ \\
$g$ & cutoff frequency of MEDOB & $500[\mathrm{rad} / \mathrm{s}]$ \\
$g_{\text {potentio }}$ & cutoff frequency of LPF for potentio & $16.8[\mathrm{rad} / \mathrm{s}]$ \\
$g_{\text {encoder }}$ & cutoff frequency of LPF for encoder & $62.8[\mathrm{rad} / \mathrm{s}]$ \\
$K_{\text {Assist }}$ & Assist gain & 1.0 \\
\hline \hline
\end{tabular}

\section{B. Torque estimation}

For the torque estimation experiment (when knee bending and stretching was performed with the leg fixed to the experimental machine), we conducted experiments in two ways, with and without feeding back the estimated torque. Parameters of the experiment are shown in Table IV. Because the resolution of the potentiometer was lower than that of the encoder, we lowered the cutoff frequency of the potentiometer's pseudo derivative. The results of experiments are shown in Figs. 7 and 8. The root mean square errors for each torque sensor value are $1.3577 \mathrm{~N} \cdot \mathrm{m}$ for no feedback and $1.1575 \mathrm{~N} \cdot \mathrm{m}$ for the case with feedback. We can confirm that the error between the estimated values and the torque sensor values are smaller than $1.4 \mathrm{~N} \cdot \mathrm{m}$. Moreover, comparing the two control mechanisms, we can confirm that the feedback control was operating with a smaller torque and that the assist control was operating effectively. However, one problem is that the estimation value at startup had a low accuracy. It is suggested that this is because the static friction and large equivalent inertia seen from the load side were not considered. Another problem is that when performing the assist control, a vibration occurs when the operation ceases. The reason given for this is that even if the user stops moving, the experimental machine itself continues to rotate due to inertia, and the tensile force works. 


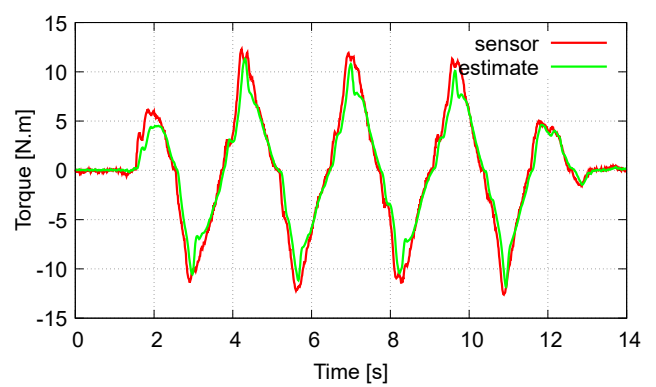

Fig. 7: Estimated torque without feedback.

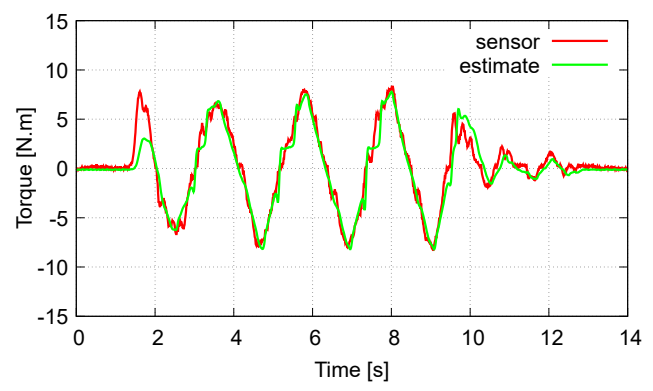

Fig. 8: Estimated torque with feedback.

\section{Motion classification}

We conducted motion classification using the nonlinear SVM. In order to accurately distinguish between the data at the time of operation and at the time of stoppage, we made separate measurements and created "teaching" data for machine learning. We used data acquired at the lowest possible operation speed and torque. Furthermore, we classified each datum into "class $=1$ " if the machine was in operation and "class $=-1$ " if it stopped. We attempted motion classification online by using the result obtained in one round of learning, but a lot of misidentifications were found. Therefore, we modified the class of misidentified data, added it to the teaching data, and completed another round of machine learning. The learning result and classification result are shown in Fig. 9 and Fig. 10, respectively. In Fig. 9, the plots are teaching data and the area shows the learning result. Orange area is that classified "in operation", and blue area is that classified "stopped".

\section{Vibration suppression}

As can be seen from Fig. 8, a small vibration occurs during stopping when assist control is applied. This section considers our attempt at vibration suppression using the classification we created, as reported in the previous section. First, Fig. 11 shows the estimated torque, angular velocity, and class when operation and stopping of the machine are repeated with constant gain. In the vibrating part, there are parts that are class $=-1$. The assist gain is classified as follows:

$$
K_{\text {Assist }}= \begin{cases}1, & \text { for class }=1 \\ 0, & \text { for class }=-1\end{cases}
$$

In the above equation, the assist control is performed as normal during machine operation, and is not performed when the

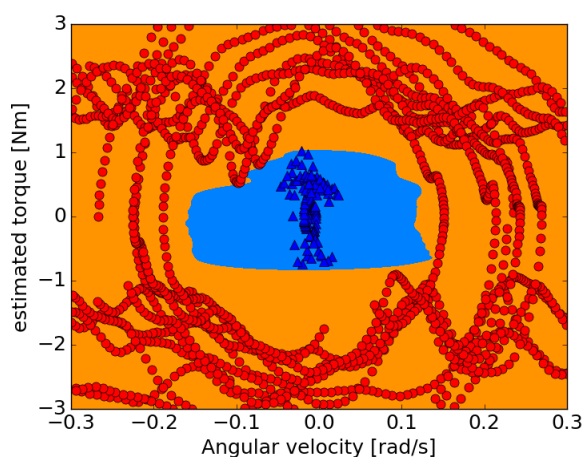

Fig. 9: Learning results (plots: teaching data, orange area: in operation, blue area: stopped).

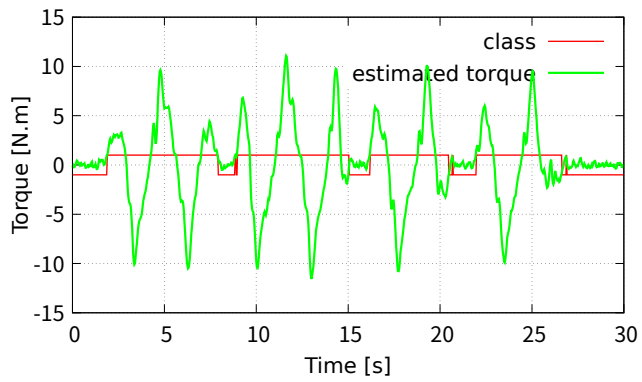

(a) Estimated torque.

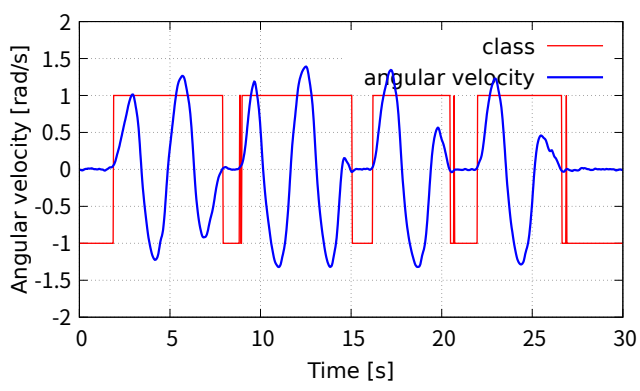

(b) Angular velocity.

Fig. 10: Classification result.

machine ceases operation. In other words, by not generating the assist torque, the motor drive is suppressed, and the vibration at the break in machine operation is reduced. Using the above conditions, we conducted the experiment again and the results are presented in Fig. 12. Comparing Fig. 11 with Fig. 12, the number of vibrations is about 4 to 5 times greater than that without assist feedback when the assist gain is constant, whereas the number of vibrations is about 1 to 2 times greater when the assist gain is changed. Therefore, it is concluded that by changing the assist gain by using motion identification, it is possible to suppress vibrations when the machine ceases operation. 


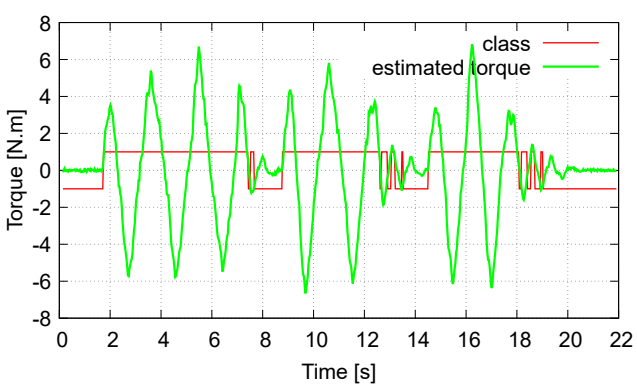

(a) Estimated torque.

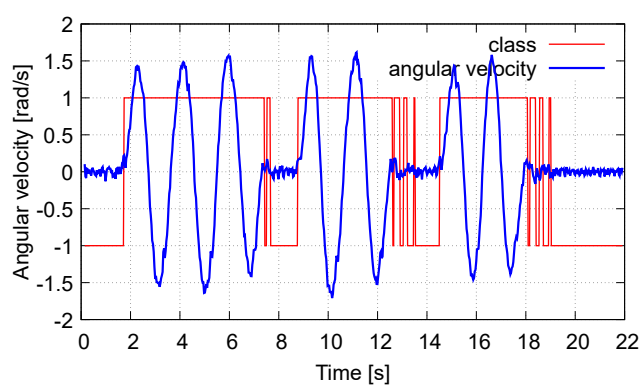

(b) Angular velocity.

Fig. 11: Result of assist gain constant.

\section{Conclusion}

In this paper, we presented the structure of our experimental machine, assist control method, and motion identification using nonlinear SVM. From the experimental results, we confirmed that the user's torque can be estimated without the requirement of a torque sensor by using a highly efficient gearbox. In addition, by changing the assist gain by using motion identification, it is possible to suppress vibrations when the machine ceases operation. From these finding, we concluded that by using a highly efficient gearbox for the powered exoskeleton, it is possible to estimate and control the force of the user without using a torque sensor.

However, in considering future improvements of the experiments, there are two main problems that need to be addressed. First, a method is required to improve estimation accuracy at startup because there is an error at the initial movement as shown in Fig. 8. Second, the motion identification was classified into only two classes ("in operation" and "stopped"), so more classifications would be required to enable a more comprehensive range of motion identifications, such as walking and crouching.

This work was supported in part by the New Energy and Industrial Technology Development Organization (NEDO) of Japan.

\section{REFERENCES}

[1] T. Kurosawa and Y. Fujimoto,"Torque sensorless control for an electric power assisted bicycle with instantaneous pedaling torque estimation," IEEJ Journal of Industry Applications, vol. 6, no. 2, pp. 124-129, 2017.

[2] T. Scaletta, S. Komada, and R. Oboe, "Development of a human assistive robot to support hip joint movement during sit-to-stand using non-linear springs," IEEJ Journal of Industry Applications, vol. 5, no. 3, pp. 261266, 2016.

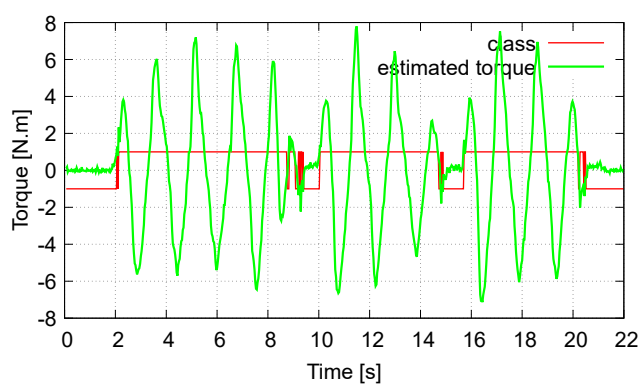

(a) Estimated torque.

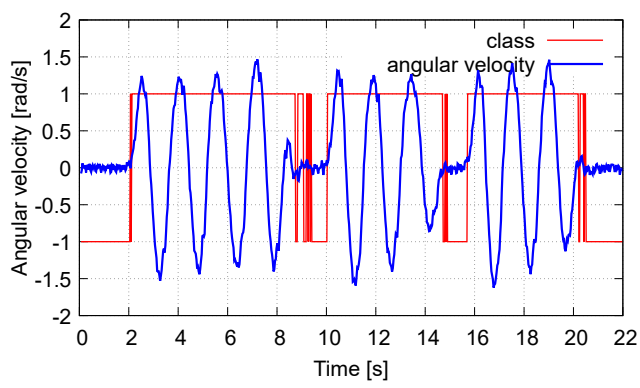

(b) Angular velocity.

Fig. 12: Result of assist gain change.

[3] H. Kawamoto and Y. Sankai, "Powered Assist System HAL-3 for Gai Disorder Person," in Proc. Int. Conf. on Computers Helping people with Special Needs (Lecture Notes on Computer Science), vol. 2398, pp. 196203, 2002.

[4] A. Zoss and H. Kazerooni, "Design of an electrically actuated lower extremity exoskeleton," Advanced Robotics, vol. 20, no. 9, pp. 967-988, 2006.

[5] T. Nef, et al., "ARMin III-arm therapy exoskeleton with an ergonomic shoulder actuation," Applied Bionics and Biomechanics, vol. 6, pp. 127142, 2009.

[6] T. Zhang, M. Tran, and H. Huang, "Design and experimental verification of hip exoskeleton with balance capacities for walking assistance," IEEE/ASME Trans. Mechatronics, vol. 23, no. 1, pp. 274-285, 2018.

[7] G. A. Pratt and M. M. Williamson, "Series Elastic Actuators", in Proc. IEEE/RSJ Int. Conf. on Intelligent Robots and Systems (IROS), vol. 1, pp. 399-406, 1995.

[8] M. Laffranchi, et all., "Development and control of a series elastic actuator equipped with a semi active friction damper for human friendly robots," Robotics and Autonomous Systems, vol. 62, no. 12, pp. 1827-1836, 2014.

[9] Y. Fujimoto and D. Kobuse, "Highly Backdrivable Robotic Actuators," in proc. IEEJ Int. Workshop on Sensing, Actuation, Motion Control, and Optimization (SAMCON), IS2-1, 2017.

[10] S. Ishikawa, M. Nishio, and T. Sugihara, "Low-cost Backdrivable Motor Control Based on Feed-forward/Feed-back Friction Compensation", in proc IEEE Int. Conf. onRobotics and Automation (ICRA), pp.5026-5031, 2015.

[11] C. Mitsantisuk, M. Nandayapa, K. Ohishi, and S. Katsura, "Design for sensorless force control of flexible robot by using resonance ratio control based on coefficient diagram method," Automatika, Journal for Control, Measurement, Electronics, Computing and Communications, vol. 54, no.1, pp. 62-73, 2013.

[12] J. A. K. Suykens and J. Vandewalle, "Least Squares Support Vector Machine Classifiers," Neural Processing Letters, no. 9, pp. 293-300, 1999.

[13] A. J. Smola and B. Sch'olkopf, "A tutorial on support vector regression," Statistics and Computing, no. 14, pp. 199-222, 2004.

[14] S. Katsura, Y. Matsumoto, and K. Ohnishi, "Modeling of Force Sensing and Validation of Disturbance Observer for Force Control," IEEE transactions on Industrial Electronics, vol. 54, no. 1, pp. 530-538, 2007. 